\title{
Training-Induced Brain Structure Changes in the Elderly
}

\author{
Janina Boyke, ${ }^{1}$ Joenna Driemeyer, ${ }^{1}$ Christian Gaser, ${ }^{2}$ Christian Büchel, ${ }^{1}$ and Arne May ${ }^{1}$ \\ ${ }^{1}$ Department of Systems Neuroscience, University of Hamburg, D-22046 Hamburg, Germany, and ${ }^{2}$ Department of Psychiatry, University of Jena, 07740 \\ Jena, Germany
}

It has been suggested that learning is associated with a transient and highly selective increase in brain gray matter in healthy young volunteers. It is not clear whether and to what extent the aging brain is still able to exhibit such structural plasticity. We built on our original study, now focusing on healthy senior citizens. We observed that elderly persons were able to learn three-ball cascade juggling, but with less proficiency compared with 20 -year-old adolescents. Similar to the young group, gray-matter changes in the older brain related to skill acquisition were observed in area hMT/V5 (middle temporal area of the visual cortex). In addition, elderly volunteers who learned to juggle showed transient increases in gray matter in the hippocampus on the left side and in the nucleus accumbens bilaterally.

Key words: age; brain; plasticity; learning; voxel-based morphometry; hippocampus

\section{Introduction}

Until quite recently, it was generally assumed that the capability of the human brain to modify its structural pattern to fit new environmental demands is restricted to the early stages of development, i.e., not beyond a specific critical period; any subsequent structural adaptations were limited to local synaptic changes. Later, based on lesion models, researchers examined the functional reorganization of cortical maps in various areas in adult brains (Weiller et al., 1993; Knecht et al., 1998; Flor et al., 2006) and provided evidence that neural systems are modifiable networks, and these processes are not limited to the early phases of development (for review, see Buonomano and Merzenich, 1998). Although cellular structures such as neurons or glial cells themselves may be highly stable and nonplastic, they are integrated into highly dynamic and plastic neural networks that adapt to environmental and intrinsic changes. Consequently, the question emerges, whether there are structural correlates of these functional adaptations. Santiago Ramón y Cajal was one of the first neuroscientists who stated that the neural architecture is not a fixed structure (DeFelipe, 2006). With the advent of sophisticated morphometric imaging techniques for investigating the structure of the human brain in vivo, several studies described activitydependent selective changes in gray matter (GM) in human adults (Maguire et al., 2000; Golestani et al., 2002; Gaser and Schlaug, 2003; Draganski et al., 2004, 2006a). Animal models have suggested that age limits the capacity for adaptive changes (Wagner et al., 2000). Aging is associated with progressive losses in function across multiple systems, including sensation, cognition, memory, motor control, and affect (Mahncke et al., 2006).

Received Feb. 18, 2008; revised April 17, 2008; accepted May 22, 2008.

This work was supported by Deutsche Forschungsgemeinschaft Grant MA 1862/2 (A.M.). We thank all volunteers for the participation in this study, Pappnase for allocating free high-performance juggling balls, and Eszter Schoell for reading this manuscript.

Correspondence should be addressed to Dr. Arne May, Assistant Professor of Neurology, Department of Systems Neuroscience, University of Hamburg, Martinistrasse 52, D-22046 Hamburg, Germany. E-mail: a.may@uke.uni-hamburg.de.

D01:10.1523/JNEUROSCI.0742-08.2008

Copyright $\odot 2008$ Society for Neuroscience $\quad$ 0270-6474/08/287031-05\$15.00/0
Clinical and experimental data suggest that perceptual motor adaptability and performance, including reaction time, speed of movement, and coordination of hands and feet, declines with advancing age (Rudisill and Toole, 1993; Kauranen and Vanharanta, 1996; Guan and Wade, 2000). Studies, both postmortem and in vivo (using imaging techniques) have shown a decline in the volume of gray matter as a result of aging (Haug and Eggers, 1991; Resnick et al., 2003). Interestingly, this decline is asymmetric and regionally accentuated (Good et al., 2001a; Hedden and Gabrieli, 2004). Considering that the brain of a 20 -year-old will change its structure when learning or practicing and that performance decreases with age, the central question arises whether the older or aged human brain structure changes in response to learning or practicing, just as the younger brain does (May and Gaser, 2006).

Focusing on the issue of whether structural neuroplasticity can arise in older people, we adopted the previously used longitudinal study design (Draganski et al., 2004) and divided a homogeneous group of 93 volunteers ( 54 female, 39 male; mean age, 60 years) matched for gender and age into two groups: training and control. Both groups were inexperienced in terms of juggling at the time point of the first scan. The subjects from the training group were given 3 months to learn classic three-ball cascade juggling. The second scan was performed at the time of skilled performance (at least $60 \mathrm{~s}$ of endurance juggling). A third scan was performed 3 months later.

\section{Materials and Methods}

Volunteers. We studied 93 healthy volunteers (54 female, 39 male; mean age, 60 years). Twenty-four participants did not complete the study, leaving 69 complete data sets with three time points. None of the volunteers could juggle. The subjects were recruited locally and they were informed that the purpose of the current study was to investigate the adaptive behavior of the CNS to learning to juggle. All of the volunteers were completely healthy; particularly, none suffered from dementia, Parkinson's disease, diabetes, or hypertension. The study received ethical approval by the local Ethics committee and written informed consent was obtained from all study participants before examination.

MRI data acquisition. All volunteers received three T1-weighted mag- 
netic resonance imaging (MRI) scans. The first scan was taken at the start of the study. Then all volunteers of the juggling group received three juggling balls and were instructed on how to learn a three-ball cascade. Following the design of our previous study (Draganski et al., 2004), the second scan was performed after 3 months, at the time of skilled performance (target: at least $60 \mathrm{~s}$ of endurance juggling), tested by one of the authors. After the second scan, none of the "jugglers" practiced their juggling skills further. A third scan was performed another 3 months later. Most participants were no longer proficient jugglers by the time of the third scan (again tested by one of the authors).

MRI was performed on a 3T MRI system (Siemens Trio) with a standard head coil. For each time point, a T1-weighted structural MRI was acquired for each subject using a three dimensional FLASH sequence (repetition time, $15 \mathrm{~ms}$; echo time, $4.9 \mathrm{~ms}$; flip angle, $25^{\circ}$; $1 \mathrm{~mm}$ slices; field of view, $256 \times 256 ; 1 \times 1 \times 1 \mathrm{~mm}$ ). All scans were visually controlled for movement artifacts. None of the T1 MRI showed any artifacts.

Voxel-based morphometry protocol. Data preprocessing and analysis were performed with SPM2 (Welcome Department of Cognitive Neurology, London, UK) running under Matlab (The MathWorks) and described in detail previously (Draganski et al., 2004; Etgen et al., 2005). In summary, preprocessing involved spatial normalization, gray-matter segmentation and $10 \mathrm{~mm}$ spatial smoothing with a Gaussian kernel. For the preprocessing steps, we registered all scans of each subject to the first scan to remove positional differences between the scans of each individual. The parameters for the following spatial normalization to the template were estimated using the first scan of each individual and then applied to the scans from all three sessions. To facilitate optimal segmentation, we estimated normalization parameters while removing nonbrain voxels (skull, sinuses) using a previously described optimized protocol (Good et al., 2001b) and a scanner- and study-specific gray-matter template. The optimized parameters, estimated while normalizing extracted GM images to the customized GM template, were reapplied to the original whole-brain images. The images aligned with the stereotactic space defined by the Montreal Neurological Institute and International Consortium for Brain Mapping (http://www.loni.ucla.edu/ICBM/) were corrected for nonuniformities in signal intensity and partitioned into gray and white matter and CSF and background (CSF) using a modified mixture model cluster analysis. Subsequently, all images were smoothed by convolving them with an isotropic Gaussian kernel of $10 \mathrm{~mm}$ full-width at half maximum.

Statistical analysis. The first analysis was a longitudinal analysis using a voxel by voxel paired $t$ test to detect regional differences in gray matter over all three time points. We tested for any regions that showed an increase as well as a decrease in brain structure between the first time point (before the learning period) compared with the second time point (after the training period), which showed an opposite pattern at time point three ( 3 months after training had stopped). We applied a threshold of $p<0.001$ (uncorrected) across the whole brain. In regions with a strong a priori hypothesis [middle temporal area of the visual cortex (hMT/V5)] (Draganski et al., 2004), small volume correction (SVC; $p<$ 0.05 ), using a sphere of $40 \mathrm{~mm}$ diameter, which covered the visual cortex, was performed.

Additionally, we performed a regression analyses using performance and exercise (hours per day) as a covariate to test for possible correlations. Again, we applied a threshold of $p<0.001$ (uncorrected).

Finally, we used a time point (before intervention, after intervention) by group (training, control) interaction analysis, testing for greater changes in the training group. The factor time point was modeled either as a transient increase (increase from time-point 1 to 2, then decrease again between time-points 2 and 3), a transient decrease (decrease from time-point 1 to 2 , then increase again between time-points 2 and 3), or a stationary change (increase or decrease from time point 1 to 2 followed by no change).

For all statistical tests, we excluded all voxels with a gray or white matter value $<0.2$ (with a maximum value of 1 ) to avoid possible edge effects around the border between gray and white matter, and to include only voxels with sufficient gray-matter proportion.

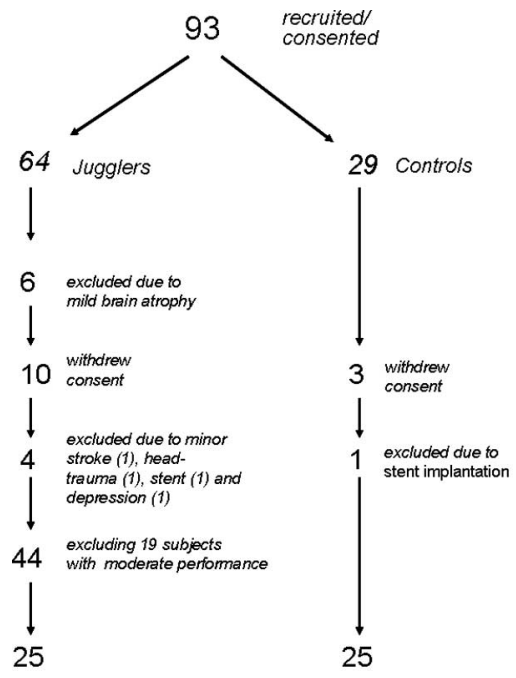

Figure 1. Flow chart of volunteer recruitment. Only 10 of 44 individuals were able to juggle $60 \mathrm{~s}$ at time point 2 . Fifteen volunteers were fluent jugglers and accomplished between 40 and $60 \mathrm{~s}$. We therefore compared the data of 25 individuals of the jugglers group who had the best results in endurance juggling (mean time, $56.7 \mathrm{~s}$ ) with the data sets of the 25 controls.

\section{Results}

Subjects

Conventional T1 MR imaging on scan 1 showed morphological abnormalities in six participants (mostly mild atrophy of the brain) and, because of methodological considerations, these participants were excluded before the exercise started. Three volunteers of the juggling group withdrew consent (three in the control group) during the study, and one participant (juggling group) suffered a minor stroke, one suffered a head trauma, one developed depression and one had to undergo coronar stenting during the study (also one in the control group). These subjects were excluded from additional analyses. This left the training group with 44 individuals ( 20 male and 24 female; 50 to 67 years old; mean age, 59.1 years) with complete data sets and the control group with 25 individuals ( 8 male and 17 female; 55 to 67 years old; mean age, 60.2 years) with complete data sets (Fig. 1). Juggling performance was less proficient than in our previous cohort of 20-year-old volunteers (Draganski et al., 2004), who accomplished at least $60 \mathrm{~s}$ of endurance juggling. Only 10 of 44 individuals in the present cohort were able to juggle $60 \mathrm{~s}$ at time point two. Another 15 volunteers were fluent jugglers and accomplished between 40 and $60 \mathrm{~s}$. Sixteen individuals were able to juggle several rounds, two individuals could only accomplish one to two rounds of a three-ball cascade, and one individual could only juggle with two balls. We therefore compared the data of 25 individuals of the jugglers group who fulfilled the defined criteria of three-ball juggling (mean time, $56.7 \mathrm{~s}$ ) with the data sets of the 25 controls. However, including the data sets of all 44 individuals of the jugglers group did not change the results, but reduced significance.

\section{Longitudinal analysis (main analysis)}

Including all time points, we found an increase in hMT/V5 on the right side $(x=25, y=-92, \mathrm{z}=-1 ; Z=4.03 ; p<0.05$, SVC) during the time of skilled performance (scan 2) compared with the first time point (scan 1). This pattern reversed when study participants were examined at time point 3 (scan 3 ). The reduction in MT gray matter in the trained old subjects was not significant compared with the baseline scan. 
Table 1. Significant changes (increase of cerebral gray matter) during the time of skilled performance (scan 2) compared with time point one

\begin{tabular}{llll}
\hline Region & $\begin{array}{l}\text { Brodmann's } \\
\text { areas }\end{array}$ & Talairach coordinates $(x, y, z)$ & $\begin{array}{l}\text { Zscore of peak } \\
\text { activation }\end{array}$ \\
\hline $\begin{array}{l}\text { Main effects analysis }(n=25) \\
\quad \text { R hMT/V5 }\end{array}$ & 18 & $25,-92,-1$ & $4.03^{*}$ \\
$\quad$ L Hippocampus & & $-18,-11,-21$ & 4.79 \\
$\quad$ Cingulate cortex & 24 & $-3,40,6$ & 4.07 \\
L Superior frontal gyrus & 10 & $-17,61,-3$ & 3.54 \\
R Gyrus precentralis & 4 & $12,-21,74$ & 4.72 \\
Interaction analysis ( $=50)$ & & & 3.21 \\
$\quad$ hMT/V5 R & 18 & $24,-91,3$ & 4.45 \\
$\quad$ L Hippocampus & & $-18,-11,-21$ & 4.79 \\
L Nucleus accumbens & & $-19,3,-14$ & 4.01 \\
R Nucleus accumbens & & $19,5,-13$ &
\end{tabular}

This pattern reversed when study participants were examined at time point 3 (after the weeks without practicing). The changes are tabulated in terms of the brain region and the corresponding Brodmann's area. The $x, y$, and $z$ coordinates are according to the Montreal Neurological Institute and International Consortium for Brain Mapping (http://www.Ioni.ucla.edu/ICBM/). Each location is the peak within a cluster (defined as the voxel with the highest Z-score). L, Left; r, right.

*Corrected for multiple comparisons (SVC).

Additionally, we found a significant change in brain gray matter $(p<0.001$, uncorrected $)$, which followed the same time pattern (increase during exercise, which receded when exercise stopped) in the left frontal cortex, the cingulate cortex, the left hippocampus, and the gyrus precentral on the right side (Table 1, Fig. 2).

All other tests (transient decrease or a stationary change) revealed no significant results.

\section{Regression analysis}

Testing for a correlation between exercise or performance and changes in gray matter revealed no significant results.

\section{Interaction analysis}

The interaction analysis of the jugglers and the controls showed that the transient increase in gray matter in the hMT/V5 area $(x=$ 24, $y=-91, z=3 ; Z=3.21 ; p<0.001$, uncorrected) was exclusively found in the training group. Additionally, only the training group, but not the controls, exhibited a transient graymatter increase (Fig. 2) in the hippocampus on the right side ( $x=$ $-18, y=-11, z=-21 ; Z=4.45 ; p<0.001$, uncorrected) and the nucleus accumbens bilaterally (right, $x=19, y=5, z=-13$; $Z=4.01$; left, $x=-19, y=3, z=-14 ; Z=4.79 ; p<0.001$, uncorrected).

\section{Discussion}

Our data suggest that, at least in principle, the human brain, even in older age, maintains its capacity to change its structure according to learning or exercise demands. Using the same motor-skillacquisition paradigm, we confirmed and extended our previous finding of transient training-induced structural brain changes for the elderly (Draganski et al., 2004) and suggest that dynamic alterations in gray matter can occur in the aged brain as well. To answer the question whether the voxel-based morphometry change in young people is similar in scale to that of the elderly, we have entered the data of the young and old subjects in a single analysis and looked for a mean effect in both samples. Although one must draw conclusions carefully, we found that on average there is a slightly smaller effect in the old subjects in the MT area, using the contrast described above (increase during exercise, which receded when exercise stopped).

Given that the older volunteers did not achieve the same level of skill as the younger subjects, these findings are all the more remarkable. Although both groups [the 20 -year-old cohort of the former study (Draganski et al., 2004) and the 60-yearold cohort of the present study] were given the same time frame ( 3 months of training) and the same instructions, only $23 \%$ of the older group achieved the target of $60 \mathrm{~s}$ endurance juggling, whereas this was the case in $100 \%$ of the younger group. Several reasons could account for this situation: Learning to juggle requires coordination control and the reciprocal transfer of visual, sensory, and motor information. Because age is correlated with weakened neuromodulatory control and noisy processing (Mahncke et al., 2006), the integration of this may be deficient, leading to poorer performance. Another possibility is an age-related decline of hand-eye coordination (Labyt et al., 2003), which might have some impact on the ability for learning a complex visuomotor skill. However, all of our volunteers had normal vision and the increase in gray matter was precisely in brain areas known for processing and storage of complex visual motion.

In addition to our finding that our cohort of 60-year-old volunteers showed the same plasticity in the same area as the brain of our previous cohort of 20 -year-old volunteers, the interaction analysis demonstrated a significant increase in gray matter in the hippocampus and the nucleus accumbens exclusively in the training group (Fig. 2). The human hippocampus (i.e., dentate gyrus) is one of only two anatomical structures known for its life-long neurogenesis (i.e., the ability to generate neurons derived from local stem cells) (Eriksson et al., 1998). Physical activity and an enriched environment (i.e., more opportunity for social interaction, physical activity, and learning) have been shown to improve the rate of adult neurogenesis and maintenance of these new neurons (Kempermann et al., 1997; Gage, 2002). A recent study by Pereira et al. (2007) demonstrating in vivo correlates of exercise-induced changes in the hippocampus confirms the theoretical possibility of angiogenesis/neurogenesis underlying plasticity processes. Although the size of the posterior hippocampal gray-matter volume has been shown to correlate positively with spatial representation skills (Maguire et al., 2000), extrahippocampal brain systems such as prefrontal gray and white matter seem to be equally involved (Moffat et al., 2007).

Focusing on this issue, we used a regression analysis involving the performance (minutes of endurance of juggling) and exercise (training minutes per day), but found no correlation between changes in gray matter and performance or exercise. We have shown previously that the posterior hippocampus shows a continuous gray-matter increase during acquisition of a great amount of highly abstract information (Draganski et al., 2006b). The present data that the hippocampus may also show a graymatter increase during a procedural learning task highlights the above-mentioned findings in mice, demonstrating increased neurogenic activity in the hippocampus during physical activity (Kempermann et al., 1997; Gage, 2002). Interestingly, these findings have been replicated in older and aged animals (Kempermann et al., 2002), suggesting that age is not per se a limiting factor for structural plasticity. Recently, Colocombe et al. (2003, 2006) demonstrated that aerobic fitness training of older humans can increase brain volume. However, it should be noted that the 


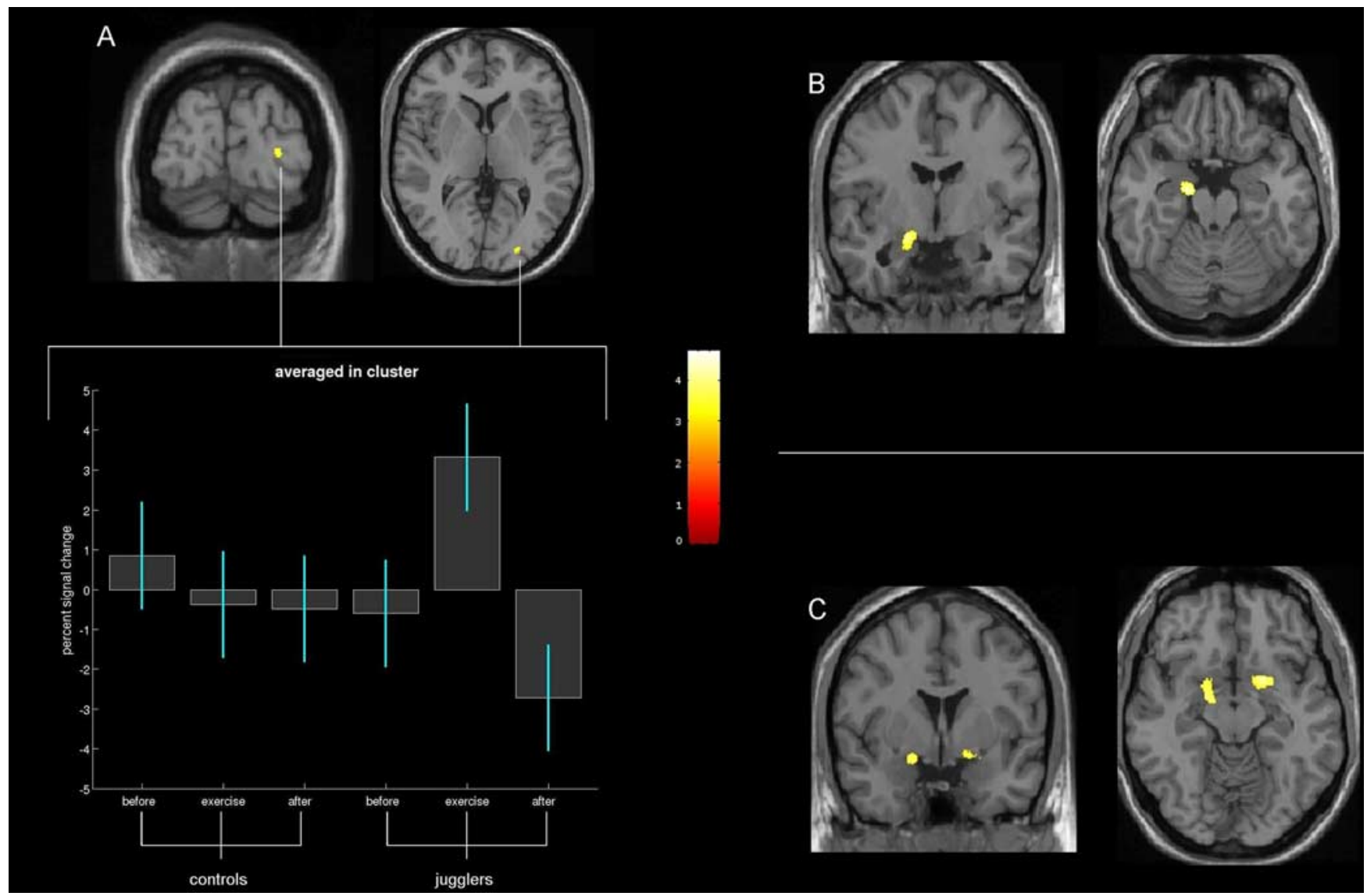

Figure 2. Time point (before intervention, after intervention) by group (jugglers, controls) interaction analysis, testing for greater changes in the jugglers group. Gray-matter increase is shown superimposed on a normalized $\mathrm{T} 1 \mathrm{image}$. The left side of the picture is the left side of the brain. $A, A$ significant gray-matter expansion was found in $\mathrm{hMT} / \mathrm{V} 5$ on the right side. This area is virtually the same area as demonstrated previously (Draganski et al., 2004). The box plot shows relative gray-matter change in the peak voxel in the right hMT for all jugglers over the three time points (error bars indicate the SD, range, and the mean for each time point). $\boldsymbol{B}, \boldsymbol{C}$, Only the jugglers group, but not the controls, showed a significant gray-matter increase in the hippocampus on the left side ( $\boldsymbol{B}$ ) and the nucleus accumbens bilaterally (C). This increase of gray matter reversed when study participants were examined at time point 3 (after the weeks without practicing).

few new neurons that are produced in the adult hippocampus cannot completely explain the structural changes detectable by MRI, suggesting that additional mechanisms must play a role. It remains intriguing, however, that adult neurogenesis occurs in a region, where we found training-induced gray-matter changes.

The gray-matter increase in the nucleus accumbens is very interesting because of its involvement in reward systems. To learn and master a new skill is certainly rewarding. The nucleus accumbens receives input from the prefrontal cortex, hippocampus, basolateral amygdala, and the ventral tegmental area, and projects to motor areas such as the ventral pallidum. It is therefore thought that the nucleus accumbens is a neural interface between limbic and motor systems, turning reward information into motivated action (Mogenson et al., 1980). Rather than structures involved in motor control, such as the cerebral cortex, cerebellum, and basal ganglia, learning to juggle seems to stimulate an increase in size of an anatomical structure that is believed to function as a "crossroad" between the limbic and the motor systems (Ikemoto and Panksepp, 1999), initiating motor responses.

Given that cortical plasticity of the human brain is, not only on a functional but also on a structural level, preserved even in later years, our data support conclusions about the potential value of exercise for elderly people (Elward and Larson, 1992; Mahncke et al., 2006). A brain-plasticity-based training program would potentially promise an improvement of the operational capabilities of aging adults (Mahncke et al., 2006; Mora et al.,
2007). As people age, they should not do less, but do more to keep and maintain their abilities.

\section{References}

Buonomano DV, Merzenich MM (1998) Cortical plasticity: from synapses to maps. Annu Rev Neurosci 21:149-186.

Colcombe SJ, Erickson KI, Raz N, Webb AG, Cohen NJ, McAuley E, Kramer AF (2003) Aerobic fitness reduces brain tissue loss in aging humans. J Gerontol A Biol Sci Med Sci 58:176-180.

Colcombe SJ, Erickson KI, Scalf PE, Kim JS, Prakash R, McAuley E, Elavsky S, Marquez DX, Hu L, Kramer AF (2006) Aerobic exercise training increases brain volume in aging humans. J Gerontol A Biol Sci Med Sci 61:1166-1170.

DeFelipe J (2006) Brain plasticity and mental processes: Cajal again. Nat Rev Neurosci 7:811-817.

Draganski B, Gaser C, Busch V, Schuierer G, Bogdahn U, May A (2004) Neuroplasticity: Changes in grey matter induced by training. Nature 427:311-312.

Draganski B, Moser T, Lummel N, Gänssbauer S, Bogdahn U, Haas F, May A (2006a) Decrease of thalamic gray matter following limb amputation. Neuroimage 31:951-957.

Draganski B, Gaser C, Kempermann G, Kuhn HG, Winkler J, Büchel C, May A (2006b) Temporal and spatial dynamics of brain structure changes during extensive learning. J Neurosci 26:6314-6317.

Elward K, Larson EB (1992) Benefits of exercise for older adults. A review of existing evidence and current recommendations for the general population. Clin Geriatr Med 8:35-50.

Eriksson PS, Perfilieva E, Björk-Eriksson T, Alborn AM, Nordborg C, Peterson DA, Gage FH (1998) Neurogenesis in the adult human hippocampus. Nat Med 4:1313-1317. 
Etgen T, Draganski B, Ilg C, Schröder M, Geisler P, Hajak G, Eisensehr I, Sander D, May A (2005) Bilateral thalamic gray matter changes in patients with restless legs syndrome. Neuroimage 24:1242-1247.

Flor H, Nikolajsen L, Staehelin Jensen T (2006) Phantom limb pain: a case of maladaptive CNS plasticity? Nat Rev Neurosci 7:873-881.

Gage FH (2002) Neurogenesis in the adult brain. J Neurosci 22:612-613.

Gaser C, Schlaug G (2003) Brain structures differ between musicians and non-musicians. J Neurosci 23:9240-9245.

Golestani N, Paus T, Zatorre RJ (2002) Anatomical correlates of learning novel speech sounds. Neuron 35:997-1010.

Good CD, Johnsrude IS, Ashburner J, Henson RN, Friston KJ, Frackowiak RS (2001a) A voxel-based morphometric study of ageing in 465 normal adult human brains. Neuroimage 14:21-36.

Good CD, Johnsrude I, Ashburner J, Henson RN, Friston KJ, Frackowiak RS (2001b) Cerebral asymmetry and the effects of sex and handedness on brain structure: a voxel-based morphometric analysis of 465 normal adult human brains. Neuroimage 14:685-700.

Guan J, Wade MG (2000) The effect of aging on adaptive eye-hand coordination. J Gerontol B Psychol Sci Soc Sci 55:P151-P162.

Haug H, Eggers R (1991) Morphometry of the human cortex cerebri and corpus striatum during aging. Neurobiol Aging 12:336-338; discussion 352-355.

Hedden T, Gabrieli JD (2004) Insights into the ageing mind: a view from cognitive neuroscience. Nat Rev Neurosci 5:87-96.

Ikemoto S, Panksepp J (1999) The role of nucleus accumbens dopamine in motivated behavior: a unifying interpretation with special reference to reward-seeking. Brain Res Brain Res Rev 31:6-41.

Kauranen K, Vanharanta H (1996) Influences of aging, gender, and handedness on motor performance of upper and lower extremities. Percept Mot Skills 82:515-525.

Kempermann G, Kuhn HG, Gage FH (1997) More hippocampal neurons in adult mice living in an enriched environment. Nature 386:493-495.

Kempermann G, Gast D, Gage FH (2002) Neuroplasticity in old age: sustained fivefold induction of hippocampal neurogenesis by long-term environmental enrichment. Ann Neurol 52:135-143.

Knecht S, Henningsen H, Höhling C, Elbert T, Flor H, Pantev C, Taub E (1998) Plasticity of plasticity? Changes in the pattern of perceptual correlates of reorganization after amputation. Brain 121:717-724.
Labyt E, Szurhaj W, Bourriez JL, Cassim F, Defebvre L, Destée A, Guieu JD, Derambure P (2003) Changes in oscillatory cortical activity related to a visuomotor task in young and elderly healthy subjects. Clin Neurophysiol 114:1153-1166.

Maguire EA, Gadian DG, Johnsrude IS, Good CD, Ashburner J, Frackowiak RS, Frith CD (2000) Navigation-related structural change in the hippocampi of taxi drivers. Proc Natl Acad Sci U S A 97:4398-4403.

Mahncke HW, Bronstone A, Merzenich MM (2006) Brain plasticity and functional losses in the aged: scientific bases for a novel intervention. Prog Brain Res 157:81-109.

May A, Gaser C (2006) Magnetic resonance-based morphometry: a window into structural plasticity of the brain. Curr Opin Neurol 19:407-411.

Moffat SD, Kennedy KM, Rodrigue KM, Raz N (2007) Extrahippocampal contributions to age differences in human spatial navigation. Cereb Cortex 17:1274-1282.

Mogenson GJ, Jones DL, Yim CY (1980) From motivation to action: functional interface between the limbic system and the motor system. Prog Neurobiol 14:69-97.

Mora F, Segovia G, Del Arco A (2007) Aging, plasticity and environmental enrichment: structural changes and neurotransmitter dynamics in several areas of the brain. Brain Res Rev 55:78-88.

Pereira AC, Huddleston DE, Brickman AM, Sosunov AA, Hen R, McKhann GM, Sloan R, Gage FH, Brown TR, Small SA (2007) An in vivo correlate of exercise-induced neurogenesis in the adult dentate gyrus. Proc Natl Acad Sci U S A 104:5638-5643.

Resnick SM, Pham DL, Kraut MA, Zonderman AB, Davatzikos C (2003) Longitudinal magnetic resonance imaging studies of older adults: a shrinking brain. J Neurosci 23:3295-3301.

Rudisill ME, Toole T (1993) Gender differences in motor performance of 50- to 79-year-old adults. Percept Mot Skills 77:939-947.

Wagner AP, Schmoll H, Badan I, Platt D, Kessler C (2000) Brain plasticity: to what extent do aged animals retain the capacity to coordinate gene activity in response to acute challenges. Exp Gerontol 35:1211-1227.

Weiller C, Ramsay SC, Wise RJ, Friston KJ, Frackowiak RS (1993) Individual patterns of functional reorganization in the human cerebral cortex after capsular infarction. Ann Neurol 33:181-189. 